\title{
CALIDAD EN LA EDUCACIÓN PERUANA, UNA EXIGENCIA SOCIAL
}

\author{
QUALITY IN PERUVIAN EDUCATION, A SOCIAL REQUIREMENT \\ Yolanda Aroquipa Durán ${ }^{*}$, (iD Reynaldo Sucari León ${ }^{1}$, (D) Nancy Chambi Condori², \\ (iD) Felipe Supo Condori ${ }^{2}$
}

\author{
yolandaaroquipa@gmail.com; reysucari@gmail.com; nany17pe@hotmail.com; felipezsupo@gmail.com \\ ${ }^{1}$ Universidad Nacional Autónoma de Huanta, Ayacucho, Perú \\ ${ }^{2}$ Universidad Nacional del Altiplano, Puno, Perú
}

*Correspondencia: Yolanda Aroquipa Durán. Email: yolandaaroquipa@gmail.com

Recibido: 26.10.19 | Aprobado: 02.12.19

\section{RESUMEN}

La sociedad actual exige una educación de calidad, voz generalizada en todo el mundo, así en el presente artículo se muestran aportes teóricos sobre las exigencias de la sociedad; hacia el MINEDU, Instituciones Educativas y los profesores, la evaluación de la calidad; muy criticada, que solo considera notas y algunas denuncias por venta de notas, también tratamos el control de la calidad; el cual exige un cambio radical en todos los agentes educativos. Concluyendo que las exigencias de la sociedad deben ser atendidas por las entidades educativas, asumiendo un compromiso de desarrollar un cambio hacia la calidad en todo nivel.

Palabras clave: Calidad, educación, exigencia social.

\begin{abstract}
The current society demands a quality education, a widespread voice throughout the world, so in this article are show theoretical contributions on the demands of society; towards the MINEDU, Educational Institutions and teachers, quality assessment; highly criticized, which only considers notes and some complaints for the sale of notes, we also deal with quality control; which demands a radical change in all educational agents. Concluding that the demands of society must be met by educational entities, assuming a commitment to develop a change towards quality at all levels.
\end{abstract}

Keywords: Quality, education, social requirement. 


\section{INTRODUCCIÓN}

En el Perú, así como en otros países del mundo se reclama y exige cada vez más una educación de calidad, la cual debe ofrecer formación para lograr una sociedad integradora y reconciliada, que solo así serán superadas las brechas de inequidad para que todos tengan oportunidades de acceso a los beneficios derivados del crecimiento y el desarrollo. Esta calidad considera los desafíos que deben asumir las universidades del mundo actual, que solo así se logrará la transformación en la educación superior para el futuro milenio. (Salas Perea, 2000)

Este crecimiento en la educación tiene una razón, el cual, es lograr el bienestar general y creciente de los estudiantes, donde las personas y las familias puedan experimentar un mejoramiento continuo de sus condiciones de vida, mientras que la sociedad en su conjunto ve incrementar y expandir sus posibilidades, oportunidades y capacidades. Frente a ello, no se puede negar que existen factores en contra; que impiden mejorar la calidad de vida de las personas. Por un lado, la realidad económica mundial y a su vez la manera de cómo el Perú se inserta en ella, por otro lado, los rasgos históricos de nuestra sociedad como la marginación, la discriminación cultual y racial, y sobre todo la pobreza. En este sentido, Arríen (1996) indica que la universidad es un hecho necesario en toda sociedad, dado que asume una responsabilidad histórica y política permitiéndole asumir desafíos en la humanidad, estos en los aspectos científico-tecnológico, económicosocial y ético-cultural.

En esta óptica, los aportes brindados por Velásquez (2014) en su propuesta indica que hoy la universidad estatal retoma acciones de calidad obviamente exigidas por el licenciamiento y la acreditación, para fortalecer los requisitos de medición, así como los de análisis y mejora de proyectos bajo la metodología de seis sigma, ello concuerda con el presente artículo ya que se propone impulsar la reflexión en torno a los procesos de mejoramiento de calidad educativa que la sociedad actual lo exige en todos los niveles de la educación, así como brindar igualdad de oportunidades para que todos los estudiantes del país logren una formación integral; en los aspectos cognitivos, en el desarrollo de habilidades y destrezas, así como su actitud para el crecimiento intelectual y personal. Para ello se han revisado varios artículos, trabajos de investigación y libros relacionados a la calidad de la educación en el Perú, materiales que brindan un aporte que permite dilucidar sobre aspectos conceptuales sobre la educación, la calidad que se da en la educación actual, métodos y sistemas de medición de la calidad educativa.

La presente revisión de literatura consta de varios aspectos como el contexto de la educación, las exigencias de la sociedad en la educación, la evaluación de la calidad y el control de la calidad. En ello se revisan varios artículos de primera fuente, así mismo se realizan algunas comparaciones sobre los conceptos recogiendo los análisis y diferentes puntos de vista de varios autores acerca de los fundamentos teóricos y experiencias, así como ventajas y desventajas. Finalmente se considera a manera de reflexión las conclusiones a las cuales se llegó luego de la revisión bibliográfica.

\section{ESTRUCTURA SOCIAL Y EDUCACIÓN}

Los gobiernos de turno de nuestro país durante su mandato modifican e imponen distintos modelos en la educación; es decir son cortoplacistas, perdiendo toda óptica a 
largo plazo, perjudicándose a los estudiantes con mayor incidencia en el sector estatal; ya sea en el sector urbano, y peor aún en el sector rural, en este sentido Alberti y Cotler (2012) indican que por lo general hay muchos trabajos referidos a la educación en la que sostienen, de forma explícita o implícita, la existencia de una relación entre estructura social y educación, resaltando que esta última se encontraría condicionada por el carácter clasista de la sociedad y por el sistema de valores de clases dominantes. Es así que, en una sociedad organizada en términos oligárquicos, que responde a una estructura social poco diferenciada, el sector "dominante" controla sin ninguna clase de interposiciones y en forma equivocada el contenido valorativo de la educación en una sociedad, imponiendo un aparato normativo que legitima su estatus de actuación.

Torres (1997) indica que, a lo largo de las tres últimas décadas, en efecto, el Banco Mundial (BM) ha venido modificando sus prioridades y, por ende, sus políticas de inversión en este sector tan vulnerable. En los años 60, los préstamos privilegiaron la infraestructura y la educación secundaria, sobre todo técnica y vocacional. En 1973 Robert Mcnamara; quien se desempeñaba como presidente del Banco Mundial, anunció un viraje radical en la política de este organismo: el BM focalizaría su acción en los más "pobres"; pero pobres en el sentido económico, atendiendo sus necesidades básicas de vivienda, salud, alimentación, agua y educación. En este último aspecto, dicho viraje se tradujo en una priorización de la escuela primaria como pilar de la estrategia para "reducir la pobreza". Así, a partir de 1970 se dio un incremento fuerte y sostenido de la inversión en educación primaria aumentándose al mismo tiempo el papel del BM en la esfera de la asistencia técnica y una reducción de los préstamos para educación secundaria. Dicho énfasis sobre la escuela primaria (y el consecuente incremento de los préstamos para este nivel) se ha visto reforzado a raíz de la Conferencia Mundial sobre "Educación para Todos", realizada en Jomtien, Tailandia, en marzo del año 1990, convocada conjuntamente por la UNESCO, UNICEF, PNUD y Banco Mundial, la cual definió la educación básica como la prioridad para dicha década y la educación primaria como la "punta de lanza" en el logro de dicha educación básica.

Esta planificación desde la década de los 90, aún no ha mejorado la educación, porque a pesar del esfuerzo iniciado décadas atrás aún son bajos los rendimientos en la educación básica, en esta línea investigadores como Cueto, Jacoby, y Pollitt (1997) presentan en sus resultados que los estudiantes de colegios privados y parroquiales obtienen puntajes altos y en el sector estatal fue y es bajo, más en el sector rural.

\section{EXIGENCIAS DE LA SOCIEDAD EN LA EDUCACIÓN}

La sociedad tiene ciertas exigencias en la educación, y esto en todos sus niveles y modalidades, principalmente y con mayor incidencia ello se está dando en el nivel superior universitario y no universitario, en tal sentido Arríen (1996) indica que la generación, producción y diseminación del conocimiento, así como la creación y el desarrollo de la ciencia, más aún la formación del capital humano, constituyen el hábitat dinámico natural de la educación superior. Este autor afirma tajantemente que en toda sociedad en la que el conocimiento alcanzó un grado relativo de independencia y especialización, ya debió resolver cómo entrenar a sus élites y formar a sus cuadros profesionales y técnicos cubriendo así la exigencia de la sociedad hacia los agentes educativos. 
Entonces nosotros nos preguntamos ¿cuáles son esas exigencias, acaso son inalcanzables?, una de ellas es que los centros de educación superior deben brindar un servicio educativo de calidad hacia los estudiantes y solo así se conseguirá formar profesionales con la capacidad de resolver problemas en esta sociedad, de lo contrario la educación se encontraría desligada de los problemas de la sociedad. Hoy en día "brindar un servicio de calidad" significa tener el "licenciamiento institucional" y ello implica cumplir con las condiciones básicas de calidad en el servicio educativo.

Respecto al concepto de calidad, Arríen (1996) menciona que el propio concepto de calidad resulta complejo y muy subjetivo, mucho más lo es cuando se relaciona con la educación (procesos y resultados, insumos y productos). Así retornando a los conceptos añejos, siempre de buen sabor, de la filosofía aristotélico-escolástica, podríamos afirmar que calidad de algo es aquello que le corresponde con su necesidad y que al faltarle afecta su naturaleza, de lo contrario no es de calidad.

Siguiendo esta línea referido a la calidad en la educación superior se tiene a Borroto Cruz y Salas Perea (1999) cuando indican que la calidad en la educación superior contemporánea está basada en una noción de cambio cualitativo, de transformación constante, utilizándose como un término de referencia de carácter comparativo, dentro de un conjunto de elementos homologables, a partir de cierto patrón o indicadores preestablecidos, siendo una resultante cualitativa de un conjunto de fuerzas impelentes, retardantes, estabilizadoras e impidientes, que tienen su origen en los diferentes grupos y posturas educativas que siempre coexisten en toda institución.

Así el mejoramiento de la calidad de la educación superior deja de ser un proceso lineal, para convertirse en un proceso multifactorial; ya que en ello intervienen muchos factores que en mayor o menor medida influyen en la calidad de la educación, cuyos resultados difieren del contexto donde es empleado, con el único propósito de incrementar la eficacia y la eficiencia en el desempeño de los agentes educativos, y en este sentido la universidad asume ese rol exigido por la sociedad, ya que allí se encuentran los académicos del más alto nivel de nuestra sociedad. (Sarabia Ramirez, 2008)

En el contexto de América Latina, González y Ayarza (1997) indican que prácticamente los países de América Latina y el Caribe el tema de la evaluación y acreditación de la educación universitaria está avanzando hacia un plano más elevado desde el punto de vista teórico, pues la UNESCO y otras organizaciones convocan a eventos donde se discuten experiencias de los países, así como los principales conceptos, en tanto, otros organismos como el Banco Mundial se interesan en este tema y se realizan intercambios y convenios entre diversos países en especial en la última década.

También Fernández (2012) menciona que la educación superior universitaria y no universitaria en América Latina está referida a un conjunto complejo de instituciones y procesos que incluso trascienden lo propiamente educativo, ya que, además de educar, dichas instituciones producen nuevos conocimientos, y prestan servicios no educativos así como la asistencia técnica al sector productivo y la ejecución de otras actividades productivas como son los centros de experimentación y producción en las universidades. 
Así como se consideran diversos aportes, la calidad en la educación superior debe guiarse de ciertos modelos o estándares, los cuales indicarán que el servicio educativo es o no de calidad. Sobre la base que la calidad es un concepto relativo asociado al proyecto institucional, CINDA-Chile ha elaborado un modelo de evaluación flexible para medir la calidad, que puede ser aplicado a instituciones de muy diversa naturaleza, con proyectos distintos y que se desenvuelven en contextos diferentes. (González y Ayarza, 1997).

En este sentido en el Perú se tiene al Sistema Nacional de Evaluación, Acreditación y Certificación de la Calidad Educativa (SINEACE) que es un conjunto de organismos, normas y procedimientos estructurados e integrados funcionalmente, destinados a definir y establecer los criterios, estándares y procesos de evaluación, acreditación y certificación a fin de asegurar los niveles básicos de calidad que debe brindar las instituciones educativas. Así mismo los órganos operadores del SINEACE son: el Concejo de Evaluación, Acreditación y Certificación de la Calidad de la Educación Superior Universitaria (CONEAU), el Concejo de Evaluación, Acreditación y Certificación de la Calidad de la Educación Superior No Universitaria (CONEACE), y el Instituto Peruano de Evaluación, Acreditación y Certificación de la Calidad de la Educación Básica (IPEBA). (SINEACE, 2012)

Para Aguila Cabrera (2005) el concepto de calidad es como excelencia, basado en la definición tradicional, para él equivale a poseer estudiantes sobresalientes, así como académicos destacados, y aseguramientos del primer nivel logrando así los objetivos. Este concepto es aplicable solo en una educación superior de elite; es decir del primer mundo, pero la educación superior latinoamericana se enfrenta al fenómeno de la masificación que es un reto que requiere una respuesta que no sea la de continuar discriminando a amplios sectores poblacionales que no forman parte de la elite, que al mismo tiempo tienen muchas necesidades sociales y económicas.

En esta línea; la calidad viene a ser el conjunto de las propiedades esenciales de algo, por las cuales ese algo se diferencia de los demás. Además, no siempre la naturaleza y propiedades de algo se hacen efectivas y reales. Se tiene calidad de manera efectiva cuando el conjunto de propiedades de algo se expresa en su forma de operación; en la medida que eso ocurra se tendrá más o menos la calidad que le es propia ya sea al servicio o al producto. Además de ello cada persona tiene un propio concepto de calidad y ello es subjetivo dado que lo que para uno es de calidad no necesariamente para otro lo es.

También Galicia (2008) indican que se conoce como calidad a todo el conjunto de cualidades que contribuyen la manera de ser de una persona o una cosa. También se identifica como el proceso seguido por una empresa de negocios para asegurarse de que sus productos o servicios cumplen con los requisitos mínimos de calidad, establecidos por la propia empresa. En otras ocasiones, se identifica la calidad con la mayor o menor satisfacción por parte de una institución o programa, de los estándares fijados por las asociaciones profesionales, o las agencias de acreditación, o de manera más simple por la satisfacción manifiesta de los empleadores. Así es necesario precisar la explosión científico - técnica de estos tiempos en el que el nuevo profesional universitario debe reunir para dar soluciones a los problemas que enfrenta la sociedad. (Salas Perea, 2000) 


\section{EVALUACIÓN Y ACREDITACIÓN ACADÉMICA}

Para Martínez (2012) la evaluación significa estimar la magnitud o la calidad de un hecho, de un proceso o producto. En consecuencia, la evaluación implica el análisis de contexto, la determinación de criterios, parámetros de referencia, variables, mediciones e indicadores, y la selección del agente evaluador. Por definición, la evaluación es relativa, está asociada a un marco conceptual y lógico de referencia, no es posible plantearla en términos absolutos.

Para el mismo Martínez una definición más operativa podría ser que la evaluación, es un proceso orientado a la toma de decisiones y a la acción, que busca determinar la pertinencia, eficiencia, efectividad, impacto y sustentabilidad del uso de recursos, actividades y resultados en función de objetivos preestablecidos o criterios definidos. Así hay muchos trabajos de investigación que sostienen que las evaluaciones de los procesos educativos son necesarios e importantes. (Zacarías, Salgado, Jiménez, y Cruz, 2014)

Adicionalmente, quizás sería útil distinguir, por su pertinencia, los siguientes conceptos: eficiencia, eficacia y efectividad. La eficiencia es la optimización de los recursos utilizados para la obtención de los resultados previstos (logro de los objetivos predefinidos). Esta noción resulta particularmente práctica ya que frecuentemente se utilizan recursos óptimamente en el logro de objetivos irrelevantes. En tanto que, la eficacia es la contribución de los resultados obtenidos al cumplimiento de objetivos globales (de la sociedad); relevancia, pertinencia, validez o utilidad socioeconómica de los resultados (objetivos predefinidos). Y por último la efectividad es la generación sistemática de resultados consistentes integrando la eficacia y la eficiencia.

Puiggrós (1996) indica que los principios de la evaluación institucional son los de conocer, comprender y explicar cómo funcionan las universidades para su mejoramiento, contribuir al perfeccionamiento de las prácticas en las universidades - en especial en lo relacionado con la toma de decisiones - y mejorar la comprensión que los actores tienen de su institución, estimulando la reflexión sobre el sentido y significado de las tareas que se realizan.

En la actualidad toda institución sea o no en el sector educativo debe y está obligado a ser evaluado; por si misma (autoevaluación) o por otras instituciones (evaluación externa), solo así se podrá garantizar la calidad en el servicio educativo. Pero ello requiere de un financiamiento el cual es muy poco en el Perú.

Respecto al problema del financiamiento de la educación pública en el Perú Saavedra y Suárez (2002) indican que entre 1970 y el 2000, la matrícula pública se expandió a una tasa promedio de 3,04\%. A pesar de esta acelerada expansión, mayor que el crecimiento de la población en edad escolar, los recursos financieros reales destinados a la educación pública se mantuvieron constantes, aunque con fluctuaciones ligadas al ciclo económico y político. También la inestabilidad macroeconómica y la imposibilidad de financiar el gasto público debido a ineficiencias institucionales y a la falta de un crecimiento sostenido de la economía determinaron que no fuera posible aumentar de manera significativa los recursos destinados a la educación. Esto, junto a una creciente presión social por incrementar la tasa de matrícula (demanda que sí fue satisfecha por el Estado), implicó una clara reducción de largo plazo en el gasto por alumno. 
Respecto a la acreditación de las instituciones universitarias Fernández (2012) menciona que la acreditación se refiere a los dictámenes requeridos para la puesta en marcha de las nuevas instituciones universitarias nacionales, para el reconocimiento de las provinciales, para la autorización provisoria de nuevas universidades privadas y para el reconocimiento definitivo de instituciones privadas con autorización provisoria.

\section{EVALUAR Y CONTROLAR LA CALIDAD}

Alvarado (1981) indica que un fenómeno está en control o es posible controlarlo cuando, con base a las experiencias pasadas, se puede predecir, al menos dentro de ciertos límites, cómo esperamos que el fenómeno va a variar en el futuro. Esta predicción significa que se puede establecer, en forma al menos aproximada, la probabilidad con la que el fenómeno observado se va a dar dentro de ciertos límites en el futuro.

Es así que teniendo en cuenta los conceptos de calidad, educación, evaluación de la calidad y control de calidad muchos autores han desarrollado diversas técnicas estadísticas para determinar los límites que indicarán que un proceso está o no controlado, que solo así se puede asegurar la calidad dentro de una organización. Para ello existen muchas técnicas, entre las más usuales se tiene a TQM y Six Sigma que primero se dieron en el mundo empresarial y con el tiempo se está extendiendo en todos los sectores, así como en la educación. (El-Khawas, 1998)

\section{Total Quality Management}

Bolívar (1999) respecto a TQM (Total Quality Management) entiende que una organización puede prosperar y sobrevivir cuando los clientes actuales y sus necesidades emergentes futuras son satisfechas por una organización de un modo más eficiente y efectivo que por sus competidoras y todo ello no excluye para nada al sector educativo. Desde esta perspectiva, cobra especial relevancia que las organizaciones recojan y analicen información sobre los clientes, por lo que los procesos de trabajo se deben dirigir a satisfacer sus necesidades y a servirlas eficientemente. Esta técnica como una estrategia de bajo coste e inversión, consiste en modificar los procesos de trabajo en modos que generen productos y servicios de alta calidad, necesarios para la supervivencia de la organización, ya sean estos en servicios o producción.

En esta línea, si se aplicara TQM a la educación superior universitaria en el Perú, se lograría buenos y excelentes resultados ya que se brindaría un servicio de calidad y de alta exigencia, generando así profesionales altamente competitivos para el desarrollo de nuestro país.

\section{Seis Sigma}

Al respecto Galicia (2008) mencionan que Seis Sigma, es una filosofía de trabajo y una estrategia de negocios, la cual se basa en el enfoque hacia el cliente, en un manejo eficiente de los datos y metodologías así como los diseños robustos, que permiten eliminar la variabilidad en los procesos y alcanzar un nivel de defectos menor o igual a 3.4 defectos por millón. Adicionalmente otros efectos obtenidos son: la reducción de los tiempos de ciclo, reducción de los costos, alta satisfacción de los clientes y lo más importante aún, efectos dramáticos en el desempeño financiero de la organización. Seis sigma, conocida también como "six sigma", no es una técnica nueva, de lo contrario es una técnica ya 
conocida que vino aplicándose en muchas organizaciones de diversos países, así otros no consideran como técnica sino de lo contrario como una filosofía.

La metodología Seis Sigma seleccionada es una estrategia de mejorar que es ampliamente utilizada con éxito en diversas empresas y su objetivo es hacer que las empresas sean más competitivas, disminuyendo la variabilidad del comportamiento de los procesos y eliminando sus desperdicios. Los fundamentos de esta metodología radican en el manejo correcto de diversas herramientas estadísticas, en nuestro caso el uso de software estadístico, por lo que es necesario contar con una formación sólida en este tipo de disciplinas, para todo directivo que está al frente de una institución educativa de nivel superior. Por otra parte, siempre se tenían presentes los factores críticos de la calidad mediante el flujo de la información y la interacción entre las personas, especialmente la interacción con el personal operativo de la empresa y sus clientes, que análogamente se puede aplicar en la educación superior. (Sarabia Ramirez, 2008)

\section{CONCLUSIONES}

Hay muchos aspectos teóricos sobre la calidad de la educación en sus diversas modalidades y niveles, tomando en cuenta otros contextos comparados con el Perú y el avance que se está dando en la actualidad respecto a la calidad del servicio educativo, mas estos conceptos sobre la calidad son subjetivos.

Una de las exigencias de la sociedad en la educación ya sea estatal o privada es que las instituciones educativas brinden un servicio educativo de calidad, considerando diversos factores, esto es entendido también por el gobierno de turno exigiendo el licenciamiento institucional a las universidades y la acreditación en el sector educativo en todos sus niveles.

Existen dos técnicas, que vienen del mundo empresarial y fácilmente son adaptados al sector educativo, estos son TQM y Six Sigma, ambas técnicas consideradas para garantizar la calidad del servicio educativo en las instituciones educativas, además con ellas se consigue la reducción del costo y tiempo en los procesos educativos.

\section{REFERENCIAS BIBLIOGRÁFICAS}

Aguila Cabrera, V. (2005). El concepto calidad en la educación universitaria: clave para el logro de la competitividad institucional. Revista Iberoamericana de Educación, 36(12), 1 7.

Alberti, G., \& Cotler, J. (2012). Aspectos sociales de la educación en el rural en el Perú. Perú Problemas, 66, 37-39.

Alvarado, R. A. (1981). Control de calidad. In Universidad Nacional Abierta y a Distancia (Vol. 4). https://doi.org/10.1016/b978-84-8086-229-5.50026-6

Arríen, J. B. (1996). Calidad y acreditación: exigencias a la universidad. Documento de Trabajo Comisión.

Bolívar, A. (1999). La educación no es un mercado. Crítica de la "Gestión de Calidad Total." Aula de Innovación Educativa, 84(83-84), 77-82. 
Borroto Cruz, R., \& Salas Perea, R. S. (1999). El reto por la calidad y la pertinencia: La evaluación desde una visión cubana. Revista Cubana de Educacion Medica Superior, 13(1), 80-91.

Cueto, S., Jacoby, E., \& Pollitt, E. (1997). Rendimiento de niños y niñas de zonas rurales y urbanas del Perú. Revista de Psicología de La PUCP, 6(12), 213-229.

El-Khawas, E. (1998). El control de calidad en la educación superior: avances recientes y dificultades por superar. Education The World Bank, 181.

Fernández, N. (2012). La evaluación y la acreditación en la Educación Superior en América Latina y El Caribe. IESALC, 66, 37-39.

Galicia, F. J. (2008). Aplicación del modelo de calidad Seis Sigma para la reducción de costos en una empresa. Instituto Politécnico Nacional.

González, L. E., \& Ayarza, H. (1997). Calidad, evaluación institucional y acreditación en la educación superior en la región Latinoamericana y del Caribe. Conferencia Regional, $1-39$.

Martínez, E. (2012). La Evaluación de la Educación Superior. Revista Actualidad Educativa, 9(2), 1-15. https://doi.org/10.22517/25395203.8111

Puiggrós, A. (1996). Educación neoliberal y quiebre educativo. Nueva Sociedad, (146), 90101.

Saavedra, J., \& Suárez, P. (2002). El financiamiento de la educación pública en el Perú: el rol de las familias. GRADE - Documento de Trabajo No 38,129.

Salas Perea, R. S. (2000). La calidad en el desarrollo profesional: avances y desafíos. Educación Médica Superior, 14(2), 136-147.

Sarabia Ramirez, E. (2008). Mejoramiento de la Gestión en Entidades Académicas de la Universidad Veracruzana. Un proyecto Seis Sigma. XI Congreso Internacional Sobre Innovaciones En Docencia e Invetigación En Ciencias Económico Administrativas.

SINEACE. (2012). Ley del Sistema Nacional de Evaluación, Acreditación y Certificación de la Calidad Educativa. In Secretaría Técncia - SINEACE (Vol. 66).

Torres, R. M. (1997). ¿ Mejorar la calidad de la educación básica? Las estrategias del Banco Mundial. Educación Al Día, 65.

Velásquez, J. M. (2014). Propuesta para aplicación de la metodología de seis sigmas en los procesos de la universidad estatal de sonora. Global Conference on Business and Finance Proceeding, 9(1), 9589.

Zacarías, J., Salgado, G., Jiménez, Y., \& Cruz, H. (2014). Calidad en la gestión de servicios. Un enfoque al servicio educativo, los casos de la mestría en Ciencias (Matemáticas ) y la Maestría en Educación Matemática de la Facultad de Ciencias Físico Matemáticas de la Benemérita Universidad Autónoma de Puebla. ECORFAN, Handbook, 257-268. 